\title{
THE NEUROSCIENCE OF VISION-BASED GRASPING a Functional Review for Computational Modeling and Bio-inspired Robotics
}

\author{
ERIS CHINELLATO and ANGEL P. DEL POBIL \\ Robotic Intelligence Lab, Jaume I University \\ Campus Riu Sec, 12071, Castellón de la Plana, Spain \\ \{eris,pobil\}@uji.es \\ Received Day Month Year \\ Revised Day Month Year
}

\begin{abstract}
The topic of vision-based grasping is being widely studied using various techniques and with different goals in humans and in other primates. The fundamental related findings are reviewed in this paper, with the aim of providing researchers from different fields, including intelligent robotics and neural computation, a comprehensive but accessible view on the subject. A detailed description of the principal sensorimotor processes and the brain areas involved in them is provided following a functional perspective, in order to make this survey especially useful for computational modeling and bio-inspired robotic applications.
\end{abstract}

Keywords: Grasping; Visual Streams; Functional modeling; Robotics.

\section{Introduction}

Robotics often aims at reproducing in artificial beings the most relevant skills characterizing animals in general and humans above all. One of the most distinctive abilities of humans, and to a minor extent of other primates, is the handling of every kind of objects in a dexterous way. Grasping and manipulating skills have been constantly pursued in robotics for their theoretical and practical implications. Nevertheless, in spite of the amount of research and technological efforts, the gap between the prehension performances of primates and that of autonomous robots is still wide, especially in unstructured, real environments.

Computational models of brain mechanisms provide an important contribution to the goal of bridging such gap. They complement neuroscience findings helping in the proposal and validation of hypothesis and theories, building on the laws of physics and chemistry, and on fundamental biological concepts such as adaptation through evolution or embodiment. On the other hand, they provide practical insights on how artificial agents, both software and hardware, can be built in order to achieve certain skills.

Bio-inspired, artificial vision-based grasping systems pursue two interconnected and complementary goals. The first goal is to obtain a unified schema of the mech- 


\section{Eris Chinellato $\&$ Angel P. del Pobil}

anisms and functionality of the brain areas most important for the planning and execution of grasping actions. Implementation of such schema on a real robotic setup allows to verify the appropriateness and plausibility level of the hypothesized mechanisms. As a second goal, those mechanisms are expected to endow robotic systems with advanced grasping capabilities not attainable with different approaches. In fact, although biomimetic robotics is a rapidly developing field, the limited literature about biological inspiration in robot grasping at cognitive level suggests that the subject has still much to offer.

The first step toward the the achievement of such ambitious goals is the thorough and extensive review of all relevant neuroscience concepts and their accurate analysis from a pragmatic, application-oriented point of view. For what concerns vision-based grasping, the most important theory that have to be taken into account is the two streams hypothesis, postulating that visual information in the brain is processed along two parallel pathways dedicated to parallel but interconnected visual and visuomotor elaborations.

The two information pathways of the primate visual cortex are called ventral stream and dorsal stream in relation to their location in the brain, depicted in Figure 1. The traditional distinction put forth by [171] and detailed by [69] talks about the ventral "what" and the dorsal "where/how" visual pathways. In fact, the ventral stream is devoted to perceptual analysis of the visual input, such as in recognition, categorization, assessment tasks. The dorsal stream is instead concerned with providing the subject the ability of interacting with its environment in a fast, effective and reliable way. This second stream is directly involved in estimating position, shape and orientation of target objects for reaching and grasping purposes.

The two cortical systems related to the visual streams were previously considered to act nearly independently [113]. Recent studies confirm that the dorsal stream is more oriented toward action-based vision, whilst the ventral one is more suitable for categorization, but their continuous interaction seems to be extremely important for allowing both of them to function properly [70]. In the manipulation of objects, although the on-line visual analysis performed by the dorsal stream is critical and strictly required for correct action execution, the contribution of the ventral stream is always very helpful and can be in some cases fundamental. For example, we could grasp a bottle even if we did not what it is. Nevertheless, if we recognize that our target object is a bottle, identify its material and assess if it is full or empty, we can reliably estimate its weight and the contact friction, and thus strongly improve the stability and reliability of our manipulation.

This paper is devoted to a detailed explanation of the concepts related to the tasks performed by the two streams, their duality and interaction, during visionbased grasping actions. Previous reviews regarding grasping have mainly focused on some aspects and certain brain regions [34], or are organized according to anatomical aspects and experimental methods [20]. This review offers a new point of view on the subject, looking at it from a functional perspective, and taking into account the 
requirements of computational modeling. Brain functions are analyzed following a logical sequence, from basic visual processing to the tactile adjustment of grasping, and paying special attention to the complementary contributions of the two streams. This approach is planned to be especially useful for modeling purposes aimed at bio-inspired robotic applications. Each subtask can be functionally modeled and associated to one or more brain areas, and the description of the areas connectivity allows to devise a wider functional organization that can be modeled at different levels of description. Some of the concepts here described were already employed and modeled, with the goal of endowing an autonomous robotic system with advanced skills in its interaction with close objects $[26,27]$.

The language we adopted in this review makes it especially suitable to be properly understood by researchers with only a basic knowledge of neuroscience concepts. The goal is to provide modelists, roboticists and other non-medical researchers with a complete review of what is actually known about the brain processes underlying vision-based grasping actions, without the need of studying complex methodologies, terminology and anatomical aspects.

Finally, it can be important to stress that this review is mainly focused on the distal components of the reach and grasp action, that is, object feature selection, grasp planning and execution. For what concerns the transport component and arm reaching issues, only briefly mentioned in this work, they are the subject of ongoing research in our group, in collaboration with leading scientists in the field [28].

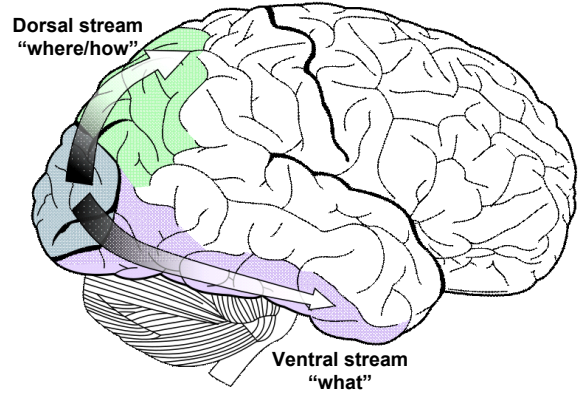

(a) Dorsal and ventral streams

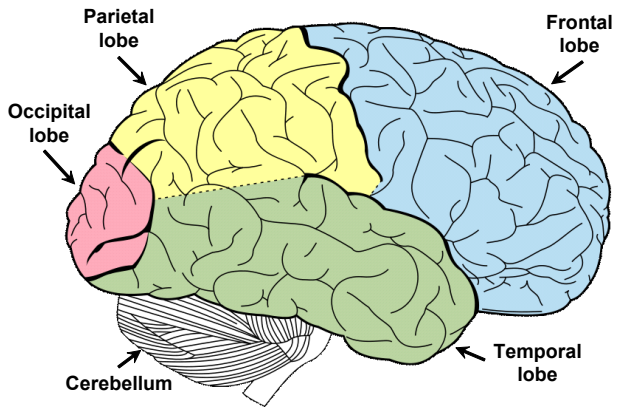

(b) Cortical lobes and cerebellum

Fig. 1. Visual streams and cortical lobes of the human brain.

\section{The two cortical streams of visual elaboration, fundamental roles and proofs of dissociation}

Looking at an object with grasping purposes activates a neural pathway which is not active when grasping actions are not involved. This activation seems to represent a potential grasping action, and is reinforced when the action is actually performed. 
Some neurons in the anterior intraparietal area (AIP) of posterior parietal cortex in monkeys are found to be active when grasping some particular objects, but also when looking at them with the purpose of grasping [146]. Some other neurons of the same area are sensitive to the size or orientation of objects, and to hand postures. Area AIP is not activated when the task is to recognize or classify objects and no practical interaction is required. A very similar pattern has been found in humans as well [33], thanks to $f M R I$ research.

In the dorsal visual stream of the primate brain, there is thus an area especially dedicated to encode the 3D features of objects in a format suitable to be used for planning and executing grasping actions. Similarly, a large part of the human brain close to the lateral-occipital sulcus (the lateral-occipital complex, LOC) is dedicated to recognize visually-presented stimuli, such as objects or faces, but is not directly involved in action execution toward them [103]. LOC is probably the most typical ventral stream area.

\subsection{A dual mechanism for vision}

The dualism between "vision for action" and "vision for perception" had been hypothesized long time before neuroimaging research [71, 112]. Studies with neurallyimpaired people, especially on two categories of brain damages, visual agnosia and optic ataxia, suggested such dualism.

Visual agnosia is the name given to a number of different disorders and syndromes in which visual object recognition is impaired [48]. Of particular interest for the two streams research is visual form agnosia, a type of agnosia that affects identification of shapes even though the subjects have preserved visual acuity, color vision, tactile recognition, and are able to move correctly and properly grasp objects presented in their peripersonal space [115, 131]. Optic ataxia occurs instead when the patient has a deficit in visually-guided arm movements that cannot be explained by motor, somatosensory, or visual acuity deficits [18, 64]. People affected by optic ataxia are unable to grasp common objects if not very clumsily and unreliably, although their recognition and classification skills are totally spared [113]. The apparent complementarity of the two impairments have been of great help for the elaboration of the two streams theory. Recent neuroimaging studies revealed that visual agnosia is caused by damages to the LOC and nearby areas, whereas damages to the dorsal stream around AIP provoke optic ataxia. For example, the brain of patient DF, suffering from visual form agnosia, does not show activation related to object identification, because her ventral stream is damaged [88]. Nevertheless, she is able to correctly perform grasping actions, and her parietal activation is rather similar to control subjects, including in the anterior intraparietal sulcus during grasping. The opposite behavioral patterns are observed in optic ataxic patients [70].

Evidence for the different role and processing mechanisms of the two pathways has been provided during the last two decades by plenty of studies following different research approaches and techniques. Recent fMRI research showed the complemen- 
tary responsiveness of the two streams in identification and spatial analysis of visual stimuli [173]. Such dissociation is confirmed for situations in which the action is observed and not directly performed by the subject [155]. Considering two of the most representative areas of the streams, AIP for the dorsal, and LOC for the ventral stream, the former shows differential activity during grasping movements with respect to reaching, whilst the latter does not. On the other hand, LOC activates whenever a recognizable object is visible (compared to scrambled images), whilst AIP only when a potentially graspable object is in view [35].

Behavioral studies based on optical illusions, distractor stimuli and concurrent tasks suggest that visual information is analyzed and processed differently by the streams [182]. According to [180], explicit object perception in the ventral stream is "scene-based" and the size and location of an object is represented contextually with the size and location of nearby objects. The control of object-directed actions by the dorsal stream follows instead an "actor-based" frame of reference, in which object location and size are represented with respect to the subject body, and especially to hand and arm. Dorsal visual analysis is driven by the absolute dimensions of the target object, and other objects in the environment are likely to be considered and hence taken into account only as potential obstacles [3]. Another distinction talks about holistic and analytical visual representations [58]: object dimensions that are perceived globally by the ventral stream are, in the same situation, processed locally by the dorsal stream if a visually-guided action is directed at the object.

Several studies (see e.g. $[79,89]$ ) demonstrated that ventral stream areas such as LOC show adaptation for different views of the same object, denoting viewpoint invariance. On the contrary, areas of the intraparietal sulcus do not exhibit such invariance, and respond to different views as they were different objects. This suggests a more "pragmatic", action-oriented on-line processing along the dorsal stream, focused on the actual situation of the environment rather than on objects' implicit quality. Even access to memory seems to be different for the two streams, and working memory related to spatial location and visual appearance is probably located in different subsystems [38].

The streams dissociation has thus been confirmed, but also criticized, by the neuroscientific community [99], and the original theory is constantly being revised and updated. The trend is toward a more integrated view of the functioning of the two streams, that have in many cases complementary tasks [72].

\subsection{Brain pathways for vision-based grasping}

The anatomy of the visual and motor cortices of human and closer superior primates is well known. Although the knowledge regarding associative regions of the brain, such as the posterior parietal or the inferior temporal cortices, is less established, it is possible to outline a simplified schema of the brain areas more directly involved in vision-based grasping actions, depicted in Figure 2. A longer list of brain areas, with acronyms or short names is provided in Table 1. Here, only an overview of the 
two pathways is given, and more details are provided in the reminder of the paper.

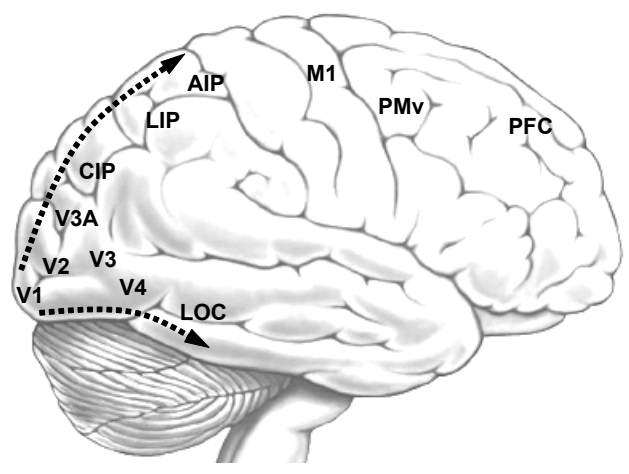

Fig. 2. Brain areas involved in vision-based grasping actions.

Visual data in primates flows from the retina to the lateral geniculate nucleus (LGN) of the thalamus, and then mainly to the primary visual cortex (V1) in the occipital lobe. The two main visual pathways go from V1 and the neighbor area V2 to the posterior parietal cortex (PPC) and the inferior temporal (IT) cortex.

Object information flowing through the ventral pathway passes through V3 and V4 to the lateral occipital complex (LOC), that is in charge of object recognition. According to the most recent interpretations of the two streams hypothesis [70], the LOC itself is implied in some action-related processing, although the way the two streams communicate is still mostly unknown.

The dorsal pathway can be further subdivided in two parallel streams concerned respectively with movement of proximal (reaching) and distal joints (grasping). The dorso-medial pathway dedicated to reaching movements includes visual area V6, visuomotor area V6A and the medial intraparietal area (MIP). The two latter areas project to the dorsal premotor cortex PMd [57]. For what concerns grasping, object related visual information flows through a dorso-lateral pathway including area V3A and the caudal intraparietal area (CIP), which extracts action-related spatial visual properties of objects. Visual data then reaches the anterior intraparietal sulcus (AIP), in which visual features are analyzed in order to plan and monitor the execution of suitable grasping actions. Motor plans are devised by parieto-frontal loops between intraparietal and premotor cortices PMd and PMv, and are sent to the primary motor cortex (M1) which release proper action execution signals.

The next sections of the paper describe concepts related to early visual processing, dorsal stream, and ventral stream processing, respectively. Exhaustive reviews of grasp-related neuroscience research are [20] and [34]. For details regarding visual areas, fundamental studies are [50] and [24]. Most brain regions cited in the text can be localized in Figure 2. 
Table 1. Principal brain areas involved in vision-based grasping. Differences between humans and other primates are discussed in the text.

\begin{tabular}{lc}
\hline \hline Brain area & Acronym \\
\hline Visual areas & \\
Primary visual cortex & V1 \\
Visual area 2 & V2 \\
Visual area 3 & V3 \\
Middle-temporal area & MT/V5 \\
\hline Dorsal stream areas & \\
Visual area 3A & V3A \\
Visual areas 6/6A & V6/V6A \\
Intraparietal sulcus & IPL \\
$\quad$ Caudal intraparietal sulcus & CIP \\
$\quad$ Anterior intraparietal sulcus & AIP \\
$\quad$ Lateral intraparietal sulcus & LIP \\
$\quad$ Medial intraparietal sulcus & MIP \\
$\quad$ Ventral intraparietal sulcus & VIP \\
Parietal reach region & PRR \\
\hline Ventral stream areas & \\
Visual area 4 & V4 \\
Lateral occipital complex & LOC \\
$\quad$ Ventral occipital temporal area & vTO \\
$\quad$ Lateral occipital cortex & LO \\
\hline Motor areas & \\
Primary motor cortex & M1 \\
Ventral premotor cortex & PMv/F5 \\
Dorsal premotor cortex & PMd \\
\hline Other areas and structures & \\
Lateral geniculate nucleus & LGN \\
Posterior parietal cortex & PPC \\
Somatosensory cortex & PFC \\
Prefrontal cortex & \\
Basal ganglia & \\
Cerebellum & \\
\hline \hline & \\
\hline
\end{tabular}

\section{Visual areas and stream separation}

The retina is the visual receptor of the human body. Visual information gathered by the retina is sent through ganglion cells to the lateral geniculate nucleus (LGN) of the thalamus. Ganglion cells are of two types: parvocellular $(\mathrm{P})$ and magnocellular $(\mathrm{M})$; the former are smaller, slower and carry many details such as color, the latter are larger and faster, and rather rough in their representations. Although these two types of cells seem to correspond nicely to the ventral and dorsal stream distinction, evidence is clearly against a simple correspondence between the subcortical and the cortical pathways, and $\mathrm{M}$ and $\mathrm{P}$ signals mix largely inside V1 [110, 51].

The LGN performs a first processing of the visual data and forwards them al- 
most entirely to the primary visual cortex (V1) in the occipital lobe [105]. Area V1, also called the striate cortex, is organized in a retinotopic manner, respecting the topological distribution of stimuli on the retina. In V1 basic visual features such as colors, bars or edges and their orientation are detected. Visual areas downstream from V1 are called extrastriate. The first extrastriate area, V2, receives most of V1 output and projects mainly to visual areas V3 and V4. Area V2 is retinotopic, has receptive fields that are larger than V1's and realizes a matching of V1 features in order to perform moderately complex visual tasks, such as detecting spatial frequencies and textures or separating foreground from background. Visual area V3 is still retinotopic and elaborates on the job of $\mathrm{V} 2$ to generate more complex invariant representations. V3 has large receptive fields and ability to detect more complex features regarding orientation, motion, depth and color of stimuli $[61,1]$. In V3 the data flow splits into the two pathways: dorsally towards the posterior parietal cortex (PPC) and ventrally to the inferior temporal (IT) cortex. Comparative studies between human and monkey (usually macaques) visual cortices reveal that their brains differ mostly in higher-order cortical regions, downstream from V3-V3A, and are more similar in lowest areas, such as V1 and V2 [174, 164, 165].

For what concerns stereoptic processing, binocular disparities are present in all visual areas, starting from V1 $[127,37,125]$. Areas V2 and V3 are increasingly capable of depth processing, in accordance with the size of their receptive fields $[4,144]$. Both in humans and monkeys, area V3A is specialized for stereoptic depth, and computes also relative disparities between pairs of visual stimuli [163, 4, 165]. Evidence regarding the role of disparity processing in visual areas is not conclusive though, as the distribution of different disparity tuning curves is rather smooth across areas [1].

Links between various disparity-selective cells allow to obtain more sophisticated response properties. For example, selectivity for absolute distance is obtained from disparities using additional information about eye position. Computation of disparity gradients is very likely performed in V3A and CIP using the outputs of many simple disparity selective cells $[1,168]$.

Visual area V5, more commonly known as the middle temporal area (MT), is very likely the most important brain region for the detection of moving visual stimuli. Both in humans and monkeys MT is selective for speed and direction of moving features [123], and its responsiveness to stereopsis signals suggests that it codes also for changes in object orientation [41, 121]. Even though the contribution of MT is required for performing grasping actions toward moving targets [150], there is no evidence for an involvement of MT in detailed 3D analysis of objects, and thus of its relevance for grasping actions toward static targets $[4,165]$.

Most projections in the visual cortex follow the described processing sequence, from V1 to higher order areas, but backprojections are widespread, and visual elaboration in the early visual areas is subject to global context influence, task requirements, and to higher order perception [105]. According to the modern view, the early 
visual cortex does not only perform the first, simple stages of visual processing, but is also involved in many higher levels of visual elaboration [176]. The visual cortex has been found to be more temporally compact than expected, and transmission times between areas spatially and hierarchically distant are very low [15]. Primary areas are thus constantly involved in all stages of visual processing, and higher areas such as MT are in a position to modulate the response of V1 and V2 neurons and suit their response to the requirement of the visual task in a reactive way. Even the LGN seems to be integrated with higher areas, and a shortcut channel of $\mathrm{M}$ cells between LGN and MT might be the instrument used by the dorsal stream to quickly separate objects from background, and bias the processing in V1-V2 [15].

\section{The action-oriented dorsal stream}

Visual region V3A can be considered as pertaining to the dorsal stream, which continues in the posterior parietal cortex, toward the top and sides of the brain. The posterior parietal cortex (PPC) is largely recognized as the main associative area of the brain dedicated to the coordination between sensory information and motor response [145]. The intraparietal sulcus (IPS) separates the superior and inferior lobes of the PPC. Several areas within and close to the IPS are dedicated to different visuomotor transformations. Many of them are described in this chapter, but special focus is put on its most posterior and anterior sections, CIP and AIP respectively.

Many of the findings explained below concern monkey data, as single cell studies allowed to collect a great deal of evidence regarding the role of intraparietal areas in macaques. Only recently, although in an ever-growing fashion, brain imaging and transcranial magnetic stimulation studies began to clarify the structure and tasks of the posterior parietal areas in humans.

Differences in control strategies depend also on structure, morphology and kinematics of body and limbs, and it is therefore very difficult to draw a full interspecies parallel [30]. The current evidence suggests that the human intraparietal cortex is more complex, and contains visuospatial processing areas that are not present, or much reduced, in monkeys [74, 122]. It has been argued that, under evolutionary pressure, parietal but not earlier regions adapted to endow humans with specific abilities, such as an improved motion-dependent 3D vision for tool manipulation $[175,122]$.

Despite the differences, a rather clear parallel between monkey and human AIP is established [76, 29]. Also, many fundamental connections correspond across species, such as the anterior intraparietal - ventral premotor and the medial intraparietal superior colliculus links [143]. Hence, it is a common procedure to consider data of similar species in order to try and work out the mechanisms behind vision-based grasping in humans $[137,74]$. Important interspecies differences are nevertheless taken into account and discussed in the following sections. 


\subsection{Posterior intraparietal sulcus}

The most posterior part of the IPS is the caudal intraparietal sulcus CIP, which is also referred to as cIPS, pIPS, PI or hCIP in the human case. Area CIP is mainly dedicated to local 3D shape and orientation processing. It receives projections from visual area V3 and V3A and is also active during visually guided grasping.

Neurons in CIP are strongly selective for the orientation of visual stimuli. Two exhaustive studies $[160,167]$ showed that selectivity toward disparity based orientation cues is predominant, but many neurons also respond (some exclusively) to perspective based orientation cues. Indeed, it seems that cue integration for obtaining better estimates of orientation is performed in this area [178]. This sort of processing by CIP neurons is the logical continuation of the simpler orientation responsiveness found in V3 and V3A. Similarly to V3A, CIP is not concerned with general purpose scene segmentation, but rather with processing the $3 \mathrm{D}$ layout of target local features $[165,168]$. In CIP, orientation of features is represented in a viewer-centered way, so that the coding is especially suitable for visuomotor transformations for reaching-grasping purposes, rather than for feature integration with the purpose of composing complex scene interpretations [149]. This is consistent with the position of CIP in a central stage of the dorsal stream. As a further proof of this, CIP does not recognize the same object seen from two different viewpoints [89].

Neurons in CIP have been found to maintain a short-term memory of 3D surface orientation [166]. This suggests a possible role of CIP in visual tracking and feature matching processes. For example, they might maintain memory of surfaces during active vision, for tracking suitable grasping surfaces.

\subsubsection{Surface orientation selective and axis orientation selective neurons}

Two main neuronal populations have been distinguished in CIP: surface orientation selective and axis orientation selective neurons. Surface orientation selective (SOS) neurons respond to a $2 \mathrm{D}$ shape in different orientations, but extract the signal of $3 \mathrm{D}$ surface orientation from a $2 \mathrm{D}$ contour viewed in a linear perspective: i.e., these neurons interpret the stimuli as the silhouette of a square plate slanted in depth [154, 149]. Experiments executed changing the proportions of the visual features showed that the responsiveness is maximum for "square" shapes, in which the two major dimensions are similar, and elongation in either width or length inhibits the response. Regarding the third, minor dimension, it seems not affecting the response up to a certain thickness, but if this threshold is overcome a clear decrease in responsiveness can be noted.

The second class of CIP neurons, axis orientation selective (AOS) neurons, represent the 3D orientation of the longitudinal axes of elongated objects. Their response increases with decreasing thickness (the two minor dimensions) and with increasing length (the major dimension), showing complementarity with SOS neurons [146]. As 
pointed out in [27] it is not clear if the reduced responsiveness with thicker objects is only due to the relative proportion between the object dimensions or also by some comparison with the hand size.

Some AOS neurons are shape selective, and distinguish for example between cylinders and square columns of similar length and thickness. This suggests that disparity gradients are used in CIP to detect also the curvature of objects [97, 119, 149]. Shape-selective AOS neurons in CIP are thus likely to maintain a prototype of 3D shape representation, that might be based on shape and curvature indexes [40].

\subsubsection{Human $C I P$}

The correspondence between monkey CIP and areas of the human intraparietal sulcus is still problematic, especially if compared with the rather well accepted interspecies matching of early visual and anterior intraparietal areas. Neuroimaging research showed nevertheless that a posterior region of the IPS activates for stimuli similar to those processed by CIP in monkeys, although human CIP seems to be located more medially in the human intraparietal sulcus than in monkeys [74]. An area located in the posterior part of IPS and clearly involved in complex orientation discrimination and coding of 3D object features have been observed [165]. The authors call it caudal parietal disparity region (CPDR), and suggest that it might be part of the human correspondent of CIP. A similar responsiveness to stereopsis defined stimuli has been registered in other studies $[153,14]$. Always using fMRI, activation in the posterior part of the human IPS has been found during orientation discrimination tasks, using both monocular and binocular stimuli [152, 119]. Although a clear correspondence is yet to be achieved, the data collected by $[152,153]$ and other studies clearly indicate that, similarly to its role in macaques, the function of human CIP is that of coding 3D features of target objects for providing AIP with the information necessary for visually-guided hand movements.

\subsubsection{CIP as a first meeting place for the two streams}

Some findings (see e.g. the work of [166]) suggest that the role of CIP might be more complex than just extracting object visual data and forwarding it to AIP. Memory related activity of CIP neurons indicates that this area might be involved in higher-order 3D visual perception. For example, visual areas V1/V4 do not have such sustained activity, whilst higher ventral stream area LOC has. There are also cues regarding direct connections between CIP and ventral stream areas. Firstly, CIP probably receives input from V4 [5], and this would be the first connection between ventral and dorsal pathways after the splitting. Moreover, some LOC neurons are selective for orientation and curvature of surfaces, but LOC receives most input from area V4, which is not sensitive to curved surfaces [124]. A link between CIP and LOC is the most likely explanation for such findings [166]. The first link, in the ventral $\rightarrow$ dorsal direction, could represent a ventral contribution to the process of 
pose and shape estimation in CIP. In fact, shape recognition allows to follow basic assumptions about objects' geometry and exploit common knowledge about the world in order to estimate size and pose of objects (e.g. to know that an object has square faces permits to use perspective in order to estimate its orientation). The dorsal $\rightarrow$ ventral link might instead accelerate object identification providing LOC with precise geometric information of local object features. Overall, 3D shape processing seems to possess a contextual nature, and on-line information is probably integrated with abstract representations in order to obtain the most likely interpretations of the visual data [162].

Summarizing, CIP has a very precise 3D orientation response, probably obtained through the integration of disparity based, stereoptic cues (prevalent) and monocular, perspective cues. Overall, a population of mixed CIP neurons, including different types of SOS and AOS neurons, is able to provide full information about 3D proportion and orientation of a target shape. This information is forwarded to AIP, where 3D orientation and shape can be coded as a unique, combined feature, and possible affordances ${ }^{\mathrm{a}}$.

\subsection{Anterior intraparietal sulcus}

The most frontal part of the IPS is the anterior intraparietal sulcus, AIP, sometimes called aIPS or, for humans, hAIP. For both monkeys and humans, AIP is largely recognized as the area of the brain dedicated to the visuomotor transformations necessary to map visual stimuli onto hand configurations suitable for grasping target objects.

Several electrophysiological studies on macaques monkeys showed that AIP activates at the visualization of a possible target object, and remains active during preshaping and manipulation [159, 148, 118]. On the contrary, AIP is not explicitly involved in spatial analysis that is not related to action: e.g., it is not active during perceptual size discrimination, for 2D pictures, or for non-graspable objects.

Different AIP neurons are tuned to different objects, to different views of the same object, and to different grips. Although some AIP neurons are specific to one spatial aspect only, similarly to CIP's, axis orientation and shape are often represented as a combined 3D feature in AIP, an probably constitute the full coding of a graspable feature [149]. Moreover, some neurons in area AIP discriminate not only between simple solid shapes, but also between complex objects composed of two or more components. According to [147], these neurons may be sensitive to very small details critical for the selection of a grip pattern.

In [118] a detailed description of experiments performed with several different conditions is provided. Neurons in AIP are found to be selectively activated according to shape (one or more of a set including ring, plate, cube, cylinder, cone

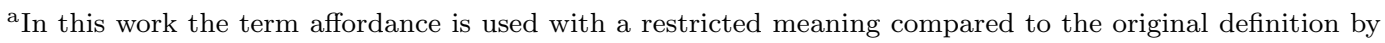
Gibson [63], to refer to a grasping possibility offered by an object to the hand. 
and sphere), size and orientation of stimuli. Different activation patterns were observed during fixation and visually-guided grasping tasks. Again, selectivity for shape/size/orientation is often merged in a combined selectivity that can be identified as a grasp configuration.

\subsubsection{Classification of AIP neurons}

Although AIP keeps active from object observation to the end of movement execution, some AIP neurons are selective for one of the following grasping sub-phases: set, preshape, enclose, hold, release [140, 42]. This subdivision is much clearer though in the premotor cortex.

A better documented classification of AIP neurons in subpopulations can be done according to their preferential response in different acting conditions [148, 118]. Three main types of AIP neurons were first classified, visual (V), visuomotor (VM) and motor (M), and the first two classes have been further subdivided into two, object $(\mathrm{O})$ and non-object $(\mathrm{NO})$, for a total of five neuronal classes:

- object type visual-dominant neurons, O-V, respond equally to simple visual presentation (fixation) of graspable $3 \mathrm{D}$ objects and during visually-guided grasping actions; these neurons show no activity during grasping in the dark or when direct view of the ongoing action is unavailable;

- non-object type visual-dominant neurons, NO-V, respond during visuallyguided grasping only, and their activation starts just before hand-object contact; they show no activation during fixation and grasping in the dark;

- object type visuomotor neurons, O-VM, are selective during fixation and during grasping actions both in the light and in the dark, but show a clear preference for visually-guided actions compared to fixation and grasping in the dark;

- non-object type visuomotor neurons, NO-VM, are selective for grasping actions both in the light and in the dark, with a clear preference for visuallyguided actions compared to grasping in the dark; they show no activation during fixation;

- motor-dominant neurons, M, are equally responsive during grasping in the light and in the dark, showing no preferential activation between the two cases; they show no activation during fixation.

On a temporal scale, object type neurons, both O-V and O-VM start their activation at the sight of the target object, and seem thus to be in charge of planning the action, transforming the spatial visual information coming from CIP into a more purely grasp-related form. At the action onset and until the contact, visuomotor neurons, both O-VM and NO-VM, reach the top of their activity, revealing a crucial role in the execution of the hand preshaping movement. Neurons NO-V and $\mathrm{M}$ also increase their activity during action execution, NO-V neurons only if vision is available, $\mathrm{M}$ neurons also in the dark. All five types of neurons remain active during the hold phase until object release, but all of them show a gradually decreasing 
activation. In the dark, only VM and M neurons stay active, and for the first the responsiveness is reduced compared to the light condition. Hence, neurons in AIP are not only dedicated to plan and begin grasping actions, but also to monitor them during their evolution.

Regarding neural coding, it seems that object type neurons ( $\mathrm{O}-\mathrm{V}$ and $\mathrm{O}-\mathrm{VM})$ describe a shape-based representation of objects, whilst motor neurons (M) code for the hand configuration suitable for grasping. Non-object type neurons (NO-V and NO-VM) maintain a someway intermediate representation. Summarizing, it appears that the classification object/non-object/motor accounts for the transformations required to pass from a visual to a motor representation of the target object. The traditional visual/visuomotor/motor classification is more likely related to temporal aspects of action execution.

\subsubsection{Human AIP}

In humans, AIP is located at the junction between anterior IPS and inferior PCS - postcentral sulcus -. Again, it is considered the most important area involved in the planning and monitoring of grasping actions. The coincidence with monkey AIP is rather uncontroversial, as many fMRI studies have been consistently showing grasping-related activation in the anterior part of the IPS for more than a decade $[9,35,22]$. For detailed reviews of such studies, refer to [21] and [170].

The most relevant difference between species is likely the absence of tactile response in macaque AIP [118], contrasted to the clear responsiveness during haptic exploration and purposive manipulation for human AIP [90, 74]. In fact, AIP is increasingly activated during multimodal processing, suggesting that it might play a specific role in cross-modal transformations of object representation between visual and tactile modalities during grasping [76]. Indeed, the current view of AIP as an associative "visual" area is probably biased by the amount of research on vision, and AIP might finally reveal itself to be as much tactile as visual [141].

In humans, AIP is preferentially activated during grasping with precision grips in comparison with full-hand power grips, suggesting a fundamental role in the fine calibration of finger positioning, as required in precision grip tasks [47, 8, 23]. AIP is probably involved also in controlling action execution by monitoring the difference between an efference copy of the motor command and visual and tactile sensory experience [132]. Various studies assign a more dynamic role to AIP beyond grasp planning. For example, transcranial magnetic stimulation of AIP ends in a clear disruption of online grasp control [65, 169], suggesting that the job of AIP is critical in the online monitoring/adjustment of hand movements.

There are also insights that AIP may execute more "cognitive" tasks and be connected to ventral stream regions. First of all, AIP and nearby areas respond to action recognition when grasping is involved $[52,155]$, indicating a more perceptual role than traditionally thought [36]. Also, a region close to the intraparietal sulcus has been found active during object recognition from non-canonical viewpoints [157]. 
The authors suggest that recognition in those cases may be supported by information regarding functional properties of the object, extracted in the CIP-AIP circuit.

Other studies mention direct connections from the inferior temporal cortex to AIP $[53,11]$ and other areas of the IPL, suggesting that AIP could use some ventral information in order to plan and execute appropriate grasping actions. Thus, after CIP, also AIP is probably connected, maybe even bidirectionally, to ventral areas, confirming the view that the collaboration between the streams is more strict than previously thought.

\subsection{Ventral premotor cortex (PMv) and other motor areas}

The motor cortex occupies the posterior half of the frontal cortex. It is composed of anterior and posterior motor areas, the former connected to the prefrontal cortex, the latter to the posterior parietal cortex. Posterior motor areas can be further subdivided in the primary motor cortex M1 (also called F1), and premotor areas F2F5. Area M1, upon reception of signals coming from the premotor cortex, activates and controls movements of specific body parts. The primary motor cortex is closely linked to corresponding areas in the primary somatosensory cortex S1, which in the case of grasping provides the tactile feedback necessary to adapt the grip to the inertial forces and the object structure [137].

A modern view of the organization, function and connectivity of the motor cortex has been proposed by [138]. The main concept is that the motor cortex is formed by a mosaic of separate areas containing independent body movement representations, which are used in motor control according to the requirements specified by corresponding areas of the posterior parietal cortex [108]. Thus, parieto-premotor connections form a series of circuits devoted to specific sensorimotor transformations. [138] define these circuits as the basic functional units of the motor system, which transform sensory information into action. According to the authors, although their hypothesis is mostly derived from monkey data, brain-imaging and anatomical evidence suggest that the same principles underlie the organization of the human motor cortex as well.

Two such circuits that have been clearly identified in monkeys connect ventral premotor areas F4 and F5 with intraparietal areas VIP and AIP respectively. The former circuit performs the sensorimotor transformations necessary for arm, neck and face movements, the latter permits the execution of hand and mouth movements, and is directly responsible for grasping actions [107]. Although their tasks are clearly related, these two circuits are described as anatomically segregated, suggesting a parallel processing between reaching and grasping actions, which are integrated only in the initial planning and the final execution phase, not in the intermediate sensorimotor transformation steps. In humans, kinematic and lesion studies support a parallel and concurrent parieto-premotor processing for reaching and grasping movements [92].

The literature description of F5 is consistent with its direct link with AIP. About 
half of F5 neurons can be considered visuomotor, as their activation begins during object fixation. Although their responsiveness is very similar to the visuomotor neurons of AIP $[117,118]$, motor specificity of F5 neurons does not depend on the object shape but on the grip used to grasp the object [129]. Additionally, neurons in F5 can code for full actions, such as grasping or pulling, and for action segments, such as preshaping or holding. Moreover, many F5 neurons are selective for one of precision grip (predominant), finger prehension or whole hand prehension [134]. According to [137], neurons in F5 code for spatial characteristics and temporal segments of grasping movements, hence constituting a vocabulary of motor prototypes to select and compose in the final action.

One of the most popularly known neuroscientific discoveries of the last decades concerns F5. This region is in fact the place where mirror neurons were observed for the first time $[45,136]$. Mirror neurons fire when the subject is performing a certain action, as normal premotor neurons of the same area, but also when the subject observes someone else performing the same action. They have been related to the ability of social interactions through understanding/prediction of other people's movements [133], to learning by imitation, and to the explanation of social behavior impairments as in autism [181].

The ventral premotor cortex (PMv) is still poorly characterized in humans. In fact, fMRI research failed to show consistent activation in the putative human equivalent of F5 during grasping movements [21]. Nevertheless, PMv is still believed to play a key role in the preparation and execution of grasping actions. TMS studies showed its importance for grasping, and its task sharing with the dorsal premotor cortex (PMd) [39]. Although the homology with macaque F5 remains controversial, there is a distinct evidence for a dissociation between PMv and PMd roles in controlling precision grasping in humans. Similarly to F5, PMv seems to perform the visuomotor transformations necessary to shape the hand to a target grip [25], whilst PMd may control the correct timing of the action [39].

\subsection{Other dorsal stream areas}

Consistently with the view of $[145,138]$ and other studies (e.g. [17]), premotorparietal circuits perform both direct and inverse coordinate transformation between vision and effector systems, to allow programming and monitoring of complex motor actions. The circuit linking AIP with F5 is not the only one necessary for the execution of accurate grasping actions, as proximal limb movements, eye and head coordination, and various posture movements are all required in order to allow the hand to perform a correct shaping sequence. Some other well-recognized areas of the posterior parietal cortex are briefly described below (for more detailed descriptions please refer to [34] and [74]).

- LIP is the lateral intraparietal area, also called parietal eye field, PEF and, for humans, hLIP or hPEF. Evidence for the role of this region in humans, and in- 
terspecies analogies are well recognized, although most studies indicate a more medial location of human LIP compared to monkey LIP. Area LIP aids in the execution of saccadic eye movements and transformation between retinotopic and head-centered coordinates [75, 151]. Neurons in LIP have been found to be modulated by both proprioceptive and retinal stimuli, suggesting that LIP contributes to distance estimation combining vergence and disparity through a gain modulation effect [119, 62].

- VIP is the ventral intraparietal area, involved in head movements coordination and near-head space analysis. Area VIP receives strong input from the motion selective area MT and responds to optic flow, detecting movements in head-center coordinates during self-motion [13]. It is also likely that VIP contributes to multimodal integration in the dorsal stream, as it is activated by visual, tactile and auditory stimuli, showing congruent receptive fields across modalities [12, 106]. Although many of its properties have been observed in human cortical areas, consistent data are not yet available for defining a clear human correspondent of monkey VIP.

- PRR is the parietal reach region, the area of the PPC dedicated to perform the reference-frame transformations and the sensorimotor coordinations necessary for pointing and reaching movements [75]. Most probably, PRR performs also a monitoring of ongoing actions and adjust them according to an efference copy of the motor signal $[96,73]$. In monkeys this area is quite well circumscribed, it includes MIP - the medial intraparietal sulcus - and visual area V6A [49]. On the other hand, pointing and reaching movements in humans seem to involve several disjunct areas of the superior parietal lobe, such as: V6A, MIP/mIPS (medial intraparietal sulcus), $\mathrm{PCu}$ (precuneus) and $\mathrm{POJ}$ (parieto-occipital junction); the exact purpose of the movement (reaching vs. pointing) and the position of the target (central vs. peripheral vision) are among the factors that differentiate the cortical activation [75].

\section{Object recognition and stream integration}

Compared to early visual areas, neurons in higher order areas such as V4 and the LOC have larger receptive fields, and can integrate information across long distances in the visual field $[80,15]$. Object areas along the ventral stream can thus represent visual stimuli with increasingly complex and invariant representations.

\subsection{The lateral occipital complex}

The lateral occipital complex (LOC) is the region of the human brain in which viewpoint invariant object representation for immediate visual recognition is performed [78]. The LOC receives high level visual input from V4 and integrates visual elements that share similar attributes of orientation, color, or depth into objects and extract them from the background [77]. Object representation in LOC is highly invariant 
with respect to the stimulus type, showing equally good performances with either 3D or silhouette images, different color maps, lightning and so on. This suggests a higher level, conceptual representation of objects, independent of the actual stimulus that allowed recognition [102].

The LOC is constituted by two different areas, anterior and posterior, which seem to maintain slightly different object representations [109]. The anterior or ventral part is called ventral temporo-occipital area (vTO/VOT, [77]), but also posterior fusiform ( $\mathrm{pFs},[100]$ ), and responds more invariantly to position and size, suggesting a volumetric 3D object representation [116]. The posterior area, the lateral occipital cortex, LO, is instead more invariant to the orientation of 2D shapes and to illumination changes. Similar subdivisions have been found in the monkey inferior temporal cortex IT [91, 60]. The occipito-temporal transition TEO, which shows a highly invariant response to object identity, is the most likely correspondent of human LOC $[177,43]$. Although these findings need to be completed and clarified, they suggest a possible mechanisms for object identification and recognition which involves both structural and image-based processing [161]. Object recognition would be achieved integrating, through feature correlation and saliency maps, a partially viewpointdependent 3D information (from vTO) with a silhouette classification performed by LO [101]. This solution would solve the long-standing issue on the nature of object recognition, confirming the validity of both the multiple view object representation model [16] and the viewpoint invariance hypothesis [89].

Thus, the assumption that ventral stream object representations should be highly viewpoint invariant would not collide with findings suggesting that in active object exploration for recognition subjects search for "preferred" views [87]. Similarly, the integrated model would partially explain why $3 \mathrm{D}$ orientation response in $\mathrm{V} 4$ - compatible with both viewpoint dependent and viewpoint invariant models [85] - is stimulus-dependent [83]. On the other hand, object recognition is very likely a gradual process rather than a binary one. [6] and [81] observed that activation in the anterior LOC is modulated by the actual level of recognition, and not by the nature of the stimulus. In any case, geometric data are integrated with additional information, regarding for example color and texture of objects, to speed up and make object recognition more reliable $[19,86]$.

Regarding possible connections of ventral stream areas with the intraparietal sulcus, a direct link has been found in the macaque brain between the most 3D responsive ventral inferior temporal area (the lower bank of the superior temporal sulcus) with CIP [91]. This link could indicate both a ventral contribution to pose estimation and a dorsal help in object recognition, as explained in Section 4.1.3.

\subsection{Other brain areas involved in grasping}

Several areas of the brain not belonging to the two streams are involved in the preparation and/or execution of vision-based grasping actions. A brief description of some very important ones is provided below. 
- Somatosensory cortex. Located in the anterior part of the parietal cortex, just behind the central sulcus, it is composed of the primary somatosensory cortex SI, the equivalent of $\mathrm{V} 1$ for the sense of touch, and the secondary somatosensory cortex SII. The former is active in correspondence to any tactile stimulation on the body, the second performs an elaboration of the sensory input in order to detect more complex patterns such as roughness, hardness, compliance estimation in hand haptic exploration $[130,120]$. Activation of somatosensory areas during grasping is consistently observed (see e.g. $[47,59,8]$ ) and, for what concerns the spatial aspects of grasping and manipulation, higher level processing of tactile information is very likely performed by AIP and nearby areas [141].

- Prefrontal cortex. The basic role of the prefrontal cortex, PFC, is the organization and orchestration of thoughts and actions in accordance with internal goals and attentional mechanisms [104]. In the specific case of grasping, the PFC is believed to mediate action selection with information on the specific task to perform [126, 95].

- Basal ganglia. Although much is still unrevealed regarding the exact function of this ancient part of the brain, the basal ganglia are probably involved in mediating between rival perceptions and/or competing motor actions. Both area AIP and the ventral premotor cortex receive inputs from the basal ganglia, but from non-overlapping regions, suggesting that they use different selection/evaluation signals that can be used in deciding among candidate target features or hand configurations in grasping [31].

- Cerebellum. The function of the cerebellum is still under debate. Nevertheless, its involvement in the coordination of action execution and in adaptive sensorimotor control is well recognized [128, 7]. In particular, it has been argued that the cerebellum is where internal forward models, largely used in sensorimotor control, are located [98]. The cerebellum has an important common feature with the posterior parietal cortex, as both play a role in sensorimotor prediction, more during action execution for the cerebellum, more planning-related for the parietal cortex [10]. Moreover, a considerable component of cerebellar output is devoted to influencing the functional operations of posterior parietal cortex, and neurons of the cerebellum that project to AIP overlap the output channel to the PMv, indicating the existence of a three-way circuit AIP-PMv-Cerebellum [31, 169].

\subsection{The visual streams in action}

The research findings described so far give a rather unequivocal view of the different tasks and processing mechanisms of the two streams. The underlying idea of the original two streams theory $[69,113]$ is that visual information has direct control over action in the dorsal stream, without any intervening mental representations. According to this view, neural activity in the dorsal stream does not reflect the representation of objects or events, but rather the direct transformation of visual information into the required coordinates for action. As stated by [93]: "object at- 
tributes are processed differently according to the task in which a subject is involved. To serve object-oriented action, these attributes are subjected to a pragmatic mode of processing, the function of which is to extract parameters that are relevant to action, and to generate the corresponding motor commands".

A basic assumption of the processing dualism is that the ventral stream makes use of a contextual coding system for size, distance and orientation of objects, while the dorsal stream needs "real-world" metrics to properly interact with the environment. The patterns of activity of LOC, AIP and other areas of the two streams strongly support this hypothesis, confirming the contrast between the conceptual and the pragmatic ways of processing of the two streams. Nevertheless, growing experimental evidence for multiple interaction between the streams cannot be disregarded, and the original theory has to be constantly updated and suited to new findings [66, 72]. For what concerns grasping, there is probably a ventral stream contribution to the grip selection process, through semantic knowledge and memories of past events [70]. Human research demonstrated that choosing a grip depends not only on its visual properties, but also on the meaning we attach to it [32] (ventral stream data) and the expected task consequent to the grip [2] (prefrontal cortex data).

As mentioned in Section 2.1, visual agnosia patient DF shows normal grasping abilities, as her dorsal stream correctly computes grasping parameters. Nevertheless, her grips on tools are functionally inappropriate, as she does not identify the target object due to her damaged ventral stream, at least until haptic exploration allows her to properly recognize the object [70]. It looks as the decision on exactly "where" to grasp the object can be taken independently from the ventral stream, but with no selection of the object feature or part more suitable for the interaction with the hand, as any semantic meaning of the action is extraneous to the dorsal stream. Moreover, DF cannot scale her grip aperture properly when she has to grasp an object that was removed from view only two seconds earlier [68]. According to the authors, this is likely due to the need of accessing object memories stored in, or accessible through, the ventral stream. Patients with optic ataxia exhibit the opposite pattern, as their grasping performances improve if a delay is introduced between target presentation and movement onset, suggesting that memory-mediated action are likely to use different mechanisms in which the dorsal pathway is less critical $[114,111]$. Psychophysical experiments on delayed grasping in different conditions with normal subjects support the idea that memory-guided grasping relies on the processing of stored information shared with the perception-based ventral system [156]. This study and related ones provide evidence that delayed grasping depends on stored memory of earlier visual information, and that the retrieval of this information shares processing resources with other cognitively demanding tasks. On the other hand, the fact that the ventral stream is able to take over the dorsal stream job as soon as the target disappears is a further demonstration of a tight integration between the streams. The question of how the control of memory-guided actions integrates the stored perceptual information with the programming of the 
action awaits further research.

\section{The third stream of visual processing}

As explained above, AIP is the cortical region in which visual information is used to code an appropriate grasping configuration for a target object, and the detailed parameters of the selected action are determined by processing in the dorsal stream. Nevertheless, action selection is very likely aided by visual processing in the ventral stream. For example, a full object description might be necessary for specifying grip and load forces through estimation or recall of the object weight. Such representation could be used also to avoid grasping objects that can not be grasped, because they are heavy, uncomfortable to handle or even dangerous [67, 179].

Considering possible different acting conditions, although some basic grasping movements may be made without the influence of the context or any top-down visual knowledge [70], in most cases parietal grasp selection is probably driven top-down by semantic information, especially for tools and well known objects [32, 54, 172]. In support of this view, [158] showed that different brain areas activate depending on the familiarity with the object, confirming that AIP elaboration is less critical if the object is well known, suggesting that in these cases the ventral stream does most of the job and the action is mainly memory-driven. Other findings [84] suggest that, although there may be a dramatic shift between the dorsal and ventral systems instantly after the target has disappeared, there also seems to be a progressive change depending on the time delay between target presentation and movement onset.

The above described interaction mechanisms between the streams might be explained by the existence of a so called "third pathway" [139]. In fact, it has been proposed that the areas of the posterior parietal cortex constituting the classical dorsal pathway should be subdivided into two different sub-systems separated by the intraparietal sulcus [53, 94]. Areas of the superior parietal lobe above the IPS, would perform the sensorimotor transformations traditionally assigned to the dorsal stream, related to the online analysis of visual data aimed at generating suitable motor reactions. The second system would contain the inferior parietal lobe, below the IPS, including regions that seem to be especially human and not matched by structures in other primate's brains [143]. These areas would be dedicated to higher level visuomotor representations, and indications for a cognitive role of AIP and nearby areas beyond the traditional pragmatic processing have been put forth by several studies [56, 25, 135, 44, 36, 82, 46, 170]. According to this view, a grasp is a sensorimotor transformation from visual information about the object to motor commands for grasping the object, but also a meaningful action that puts in relation the agent with a feature of the environment. The new, ventro-dorsal stream of the inferior parietal lobe would constitute an ideal convergence focus for the integration of conceptual ventral information with traditional online dorsal data [142, 55]. More research is though needed to assess and develop this hypothesis. 


\section{Conclusions}

We have reviewed the role and connectivity of the brain areas most critical for the correct execution of vision-based grasping actions. Our review was conducted with the aim of clarifying what tasks are performed by each area and how tasks and areas are logically related. It remained clear how the posterior parietal - premotor cortical loop is fundamental in reaching and grasping actions, and how other areas, especially of the ventral stream, contribute to optimal and versatile action planning. Brain mechanisms have been described in this review considering a functional point of view, especially suitable for modeling purposes, aimed at the application of neuroscience concepts to autonomous robots, and to the validation of brain theories through tests on artificial agents. New research in neuroscience, artificial intelligence and bio-inspired robotics will help in shedding further light on the brain processes that endow primates, and especially humans, with such outstanding manipulation skills.

\section{Acknowledgments}

Support for this research has been provided in part by the European Commission's Seventh Framework Programme FP7/2007-2013 under grant agreement 217077 (EYESHOTS project), by Ministerio de Ciencia y Innovación (DPI-2008-06636) and by Fundació Caixa-Castello-Bancaixa (P1-1B2008-51). The authors would like to thank Mel Goodale and Jody Culham for the many helpful discussions and patient tutorials on grasping, Umberto Castiello and Caterina Ansuini for comments on earlier drafts of this paper, and the anonymous reviewers for their detailed and pertinent comments and advice.

\section{References}

[1] D. L. Adams and S. Zeki. Functional organization of macaque V3 for stereoscopic depth. Journal of Neurophysiology, 86(5):2195-2203, November 2001.

[2] C. Ansuini, M. Santello, S. Massaccesi, and U. Castiello. Effects of end-goal on hand shaping. Journal of Neurophysiology, 95(4):2456-2465, April 2006.

[3] C. Ansuini, V. Tognin, L. Turella, and U. Castiello. Distractor objects affect fingers' angular distances but not fingers' shaping during grasping. Experimental Brain Research, 178(2):194-205, April 2007.

[4] B. T. Backus, D. J. Fleet, A. J. Parker, and D. J. Heeger. Human cortical activity correlates with stereoscopic depth perception. Journal of Neurophysiology, 86(4):20542068, October 2001.

[5] J. S. Baizer, L. G. Ungerleider, and R. Desimone. Organization of visual inputs to the inferior temporal and posterior parietal cortex in macaques. Journal of Neuroscience, 11(1):168-190, January 1991.

[6] M. Bar, R. B. Tootell, D. L. Schacter, D. N. Greve, B. Fischl, J. D. Mendola, B. R. Rosen, and A. M. Dale. Cortical mechanisms specific to explicit visual object recognition. Neuron, 29(2):529-535, February 2001. 
[7] J. S. Barlow. The cerebellum and adaptive control. Cambridge University Press, 2002.

[8] C. Begliomini, M. B. Wall, A. T. Smith, and U. Castiello. Differential cortical activity for precision and whole-hand visually guided grasping in humans. European Journal of Neuroscience, 25(4):1245-1252, February 2007.

[9] F. Binkofski, C. Dohle, S. Posse, K. M. Stephan, H. Hefter, R. J. Seitz, and H. J. Freund. Human anterior intraparietal area subserves prehension: a combined lesion and functional MRI activation study. Neurology, 50(5):1253-1259, May 1998.

[10] S.-J. Blakemore and A. Sirigu. Action prediction in the cerebellum and in the parietal lobe. Experimental Brain Research, 153(2):239-245, November 2003.

[11] E. Borra, A. Belmalih, R. Calzavara, M. Gerbella, A. Murata, S. Rozzi, and G. Luppino. Cortical connections of the macaque anterior intraparietal (AIP) area. Cerebral Cortex, 18(5):1094-1111, August 2007.

[12] F. Bremmer, F. Klam, J.-R. Duhamel, S. Ben Hamed, and W. Graf. Visual-vestibular interactive responses in the macaque ventral intraparietal area (VIP). European Journal of Neuroscience, 16(8):1569-1586, October 2002.

[13] F. Bremmer, A. Schlack, J. R. Duhamel, W. Graf, and G. R. Fink. Space coding in primate posterior parietal cortex. Neuroimage, 14(1 Pt 2):S46-S51, July 2001.

[14] G. J. Brouwer, R. van Ee, and J. Schwarzbach. Activation in visual cortex correlates with the awareness of stereoscopic depth. Journal of Neuroscience, 25(45):1040310413, November 2005.

[15] J. Bullier. Integrated model of visual processing. Brain Research Reviews, 36(2-3):96107, October 2001.

[16] H. H. Bülthoff, S. Y. Edelman, and M. J. Tarr. How are three-dimensional objects represented in the brain? Cerebral Cortex, 5(3):247-260, 1995.

[17] C. A. Buneo and R. A. Andersen. The posterior parietal cortex: sensorimotor interface for the planning and online control of visually guided movements. Neuropsychologia, 44(13):2594-2606, 2006.

[18] L. Buxbaum and H. Branch Coslett. Subtypes of optic ataxia: Reframing the disconnection account. Neurocase, 3:159-166, 1997.

[19] J. S. Cant, S. R. Arnott, and M. A. Goodale. fmr-adaptation reveals separate processing regions for the perception of form and texture in the human ventral stream. Exp Brain Res, 192(3):391-405, Jan 2009.

[20] U. Castiello. The neuroscience of grasping. Nature Reviews Neuroscience, 6(9):726$736,2005$.

[21] U. Castiello and C. Begliomini. The cortical control of visually guided grasping. Neuroscientist, 14(2):157-170, April 2008.

[22] C. Cavina-Pratesi, M. A. Goodale, and J. C. Culham. fMRI reveals a dissociation between grasping and perceiving the size of real 3D objects. PLoS ONE, 2(5):e424, 2007.

[23] C. Cavina-Pratesi, S. Monaco, T. McAdam, D. Milner, T. Schenk, and J. C. Culham. Which aspects of hand-preshaping does human AIP compute during visually guided actions? Evidence from event-related fMRI. In annual meeting of the Society for Neuroscience, 2007.

[24] L. M. Chalupa and J. S. Werner, editors. The visual neurosciences. MIT Press, 2003.

[25] L. L. Chao and A. Martin. Representation of manipulable man-made objects in the 
dorsal stream. Neuroimage, 12(4):478-484, October 2000.

[26] E. Chinellato and A. P. del Pobil. Distance and orientation estimation of graspable objects in natural and artificial systems. Neurocomputing, 72:879-886, 2008.

[27] E. Chinellato and A. P. del Pobil. Neural coding in the dorsal visual stream. In International Conference on the Simulation of Adaptive Behavior, 2008.

[28] E. Chinellato, B. J. Grzyb, N. Marzocchi, A. Bosco, P. Fattori, and A. P. del Pobil. Eye-hand coordination for reaching in dorsal stream area V6A: Computational lessons. In LNCS. Springer, 2009.

[29] H.-J. Choi, K. Zilles, H. Mohlberg, A. Schleicher, G. R. Fink, E. Armstrong, and K. Amunts. Cytoarchitectonic identification and probabilistic mapping of two distinct areas within the anterior ventral bank of the human intraparietal sulcus. The Journal of Comparative Neurology, 495(1):53-69, 2006.

[30] M. I. Christel and A. Billard. Comparison between macaques' and humans' kinematics of prehension: the role of morphological differences and control mechanisms. Behavioural Brain Research, 131(1-2):169-184, April 2002.

[31] D. M. Clower, R. P. Dum, and P. L. Strick. Basal ganglia and cerebellar inputs to 'AIP'. Cerebral Cortex, 15(7):913-920, July 2005.

[32] S. H. Creem and D. R. Proffitt. Grasping objects by their handles: a necessary interaction between cognition and action. Journal of Experimental Psychology: Human Perception and Performance, 27(1):218-228, February 2001.

[33] J. C. Culham. Human brain imaging reveals a parietal area specialized for grasping. In N. Kanwisher and J. Duncan, editors, Functional Neuroimaging of Visual Cognition: Attention and Performance XX, pages 417-438. Oxford University Press, 2004.

[34] J. C. Culham, C. Cavina-Pratesi, and A. Singhal. The role of parietal cortex in visuomotor control: what have we learned from neuroimaging? Neuropsychologia, 44(13):2668-2684, 2006.

[35] J. C. Culham, S. L. Danckert, J. F. X. DeSouza, J. S. Gati, R. S. Menon, and M. A. Goodale. Visually guided grasping produces fMRI activation in dorsal but not ventral stream brain areas. Experimental Brain Research, 153(2):180-189, November 2003.

[36] J. C. Culham and K. F. Valyear. Human parietal cortex in action. Current Opinion in Neurobiology, 16(2):205-212, April 2006.

[37] B. G. Cumming and G. C. DeAngelis. The physiology of stereopsis. Annual Review of Neuroscience, 24:203-238, 2001.

[38] S. Darling, S. Della Sala, R. H. Logie, and A. Cantagallo. Neuropsychological evidence for separating components of visuospatial working memory. Journal of Neurology, 253(2):176 - 180, February 2006.

[39] M. Davare, M. Andres, G.Cosnard, J.-L. Thonnard, and E. Olivier. Dissociating the role of ventral and dorsal premotor cortex in precision grasping. Journal of Neuroscience, 26(8):2260-2268, February 2006.

[40] S. C. de Vries, A. M. Kappers, and J. J. Koenderink. Influence of surface attitude and curvature scaling on discrimination of binocularly presented curved surfaces. Vision Research, 34(18):2409-2423, September 1994.

[41] G. C. DeAngelis, B. G. Cumming, and W. T. Newsome. Cortical area MT and the perception of stereoscopic depth. Nature, 394(6694):677-680, August 1998.

[42] D. J. Debowy, S. Ghosh, J. Y. Ro, and E. P. Gardner. Comparison of neuronal firing 
rates in somatosensory and posterior parietal cortex during prehension. Experimental Brain Research, 137(3-4):269-291, April 2001.

[43] K. Denys, W. Vanduffel, D. Fize, K. Nelissen, H. Peuskens, D. Van Essen, and G. A. Orban. The processing of visual shape in the cerebral cortex of human and nonhuman primates: a functional magnetic resonance imaging study. Journal of Neuroscience, 24(10):2551-2565, March 2004.

[44] N. Derbyshire, R. Ellis, and M. Tucker. The potentiation of two components of the reach-to-grasp action during object categorisation in visual memory. ACTA Psychologica (Amsterdam), 122:74-98, December 2005.

[45] G. Di Pellegrino, L. Fadiga, L. Fogassi, V. Gallese, and G. Rizzolatti. Understanding motor events: a neurophysiological study. Experimental Brain Research, 91(1):176180, 1992.

[46] J.-B. Durand, K. Nelissen, O. Joly, C. Wardak, J. T. Todd, J. F. Norman, P. Janssen, W. Vanduffel, and G. A. Orban. Anterior regions of monkey parietal cortex process visual 3D shape. Neuron, 55(3):493-505, August 2007.

[47] H. H. Ehrsson, A. Fagergren, T. Jonsson, G. Westling, R. S. Johansson, and H. Forssberg. Cortical activity in precision- versus power-grip tasks: an fMRI study. Journal of Neurophysiology, 83(1):528-536, January 2000.

[48] M. J. Farah. Visual agnosia. MIT Press, 2004.

[49] P. Fattori, D. F. Kutz, R. Breveglieri, N. Marzocchi, and C. Galletti. Spatial tuning of reaching activity in the medial parieto-occipital cortex (area v6a) of macaque monkey. Eur J Neurosci, 22(4):956-972, Aug 2005.

[50] D. J. Felleman and D. C. Van Essen. Distributed hierarchical processing in the primate cerebral cortex. Cerebral Cortex, 1(1):1-47, 1991.

[51] V. P. Ferrera, T. A. Nealey, and J. H. Maunsell. Mixed parvocellular and magnocellular geniculate signals in visual area V4. Nature, 358(6389):756-761, August 1992.

[52] L. Fogassi, P. F. Ferrari, B. Gesierich, S. Rozzi, F. Chersi, and G. Rizzolatti. Parietal lobe: from action organization to intention understanding. Science, 308(5722):662667, April 2005.

[53] L. Fogassi and G. Luppino. Motor functions of the parietal lobe. Current Opinion in Neurobiology, 15(6):626-631, December 2005.

[54] S. H. Frey, D. Vinton, R. Norlund, and S. T. Grafton. Cortical topography of human anterior intraparietal cortex active during visually guided grasping. Cognitive Brain Research, 23(2-3):397-405, May 2005.

[55] V. Gallese. The "conscious" dorsal stream: Embodied simulation and its role in space and action conscious awareness. Psyche, 13(1), April 2007.

[56] V. Gallese, L. Craighero, L. Fadiga, and L. Fogassi. Perception through action. Psyche, $5(21): 1,1999$.

[57] C. Galletti, D. F. Kutz, M. Gamberini, R. Breveglieri, and P. Fattori. Role of the medial parieto-occipital cortex in the control of reaching and grasping movements. Experimental Brain Research, 153(2):158-170, November 2003.

[58] T. Ganel and M. A. Goodale. Visual control of action but not perception requires analytical processing of object shape. Nature, 426(6967):664-667, December 2003.

[59] E. P. Gardner, D. J. Debowy, J. Y. Ro, S. Ghosh, and K. Srinivasa Babu. Sensory monitoring of prehension in the parietal lobe: a study using digital video. Behavioural 
Brain Research, 135(1-2):213-224, September 2002.

[60] R. Gattass, S. Nascimento-Silva, J. G. M. Soares, B. Lima, A. K. Jansen, A. C. M. Diogo, M. F. Farias, M. M. Botelho, P. Elia, O. S. Mariani, J. Azzi, and M. Fiorani. Cortical visual areas in monkeys: location, topography, connections, columns, plasticity and cortical dynamics. Philosophical Transactions of the Royal Society B: Biological Sciences, 360(1456):709-731, April 2005.

[61] K.R. Gegenfurtner, D.C. Kiper, and J.B. Levitt. Functional properties of neurons in macaque area V3. Journal of Neurophysiology, 77(4):1906-1923, April 1997.

[62] A. Genovesio and S. Ferraina. Integration of retinal disparity and fixation-distance related signals toward an egocentric coding of distance in the posterior parietal cortex of primates. Journal of Neurophysiology, 91(6):2670-2684, June 2004.

[63] J. J. Gibson. The ecological approach to visual perception. Lawrence Erlbaum Associates, New Jersey, USA, 1979.

[64] S. Glover. Optic ataxia as a deficit specific to the on-line control of actions. Neuroscience $\& 3$ Biobehavioral Reviews, 27(5):447-456, August 2003.

[65] S. Glover, R. C. Miall, and M. F. S. Rushworth. Parietal rTMS disrupts the initiation but not the execution of on-line adjustments to a perturbation of object size. The Journal of Cognitive Neuroscience, 17(1):124-136, January 2005.

[66] M. A. Goodale and A. M. Haffenden. Interactions between the dorsal and ventral streams of visual processing. Advances in Neurology, 93:249-267, 2003.

[67] M. A. Goodale and G. K. Humphrey. The objects of action and perception. Cognition, 67(1-2):181-207, July 1998.

[68] M. A. Goodale, J. P. Meenan, H. H. Bülthoff, D. A. Nicolle, K. J. Murphy, and C. I. Racicot. Separate neural pathways for the visual analysis of object shape in perception and prehension. Current Biology, 4(7):604-610, July 1994.

[69] M. A. Goodale and A. D. Milner. Separate visual pathways for perception and action. Trends in Neurosciences, 15(1):20-25, January 1992.

[70] M. A. Goodale and A. D. Milner. Sight Unseen. Oxford University Press, 2004.

[71] M. A. Goodale, A. D. Milner, L. S. Jakobson, and D. P. Carey. A neurological dissociation between perceiving objects and grasping them. Nature, 349(6305):154-156, January 1991.

[72] M. A. Goodale and D. A. Westwood. An evolving view of duplex vision: separate but interacting cortical pathways for perception and action. Current Opinion in Neurobiology, 14(2):203-211, April 2004.

[73] H. Gréa, L. Pisella, Y. Rossetti, M. Desmurget, C. Tilikete, S. Grafton, C. Prablanc, and A. Vighetto. A lesion of the posterior parietal cortex disrupts on-line adjustments during aiming movements. Neuropsychologia, 40(13):2471-2480, 2002.

[74] C. Grefkes and G. R. Fink. The functional organization of the intraparietal sulcus in humans and monkeys. Journal of Anatomy, 207(1):3-17, July 2005.

[75] C. Grefkes, A. Ritzl, K. Zilles, and G. R. Fink. Human medial intraparietal cortex subserves visuomotor coordinate transformation. Neuroimage, 23(4):1494-1506, December 2004.

[76] C. Grefkes, P. H. Weiss, K. Zilles, and G. R. Fink. Crossmodal processing of object features in human anterior intraparietal cortex: an fMRI study implies equivalencies between humans and monkeys. Neuron, 35(1):173-184, July 2002. 
[77] K. Grill-Spector. The neural basis of object perception. Current Opinion in Neurobiology, 13(2):159-166, April 2003.

[78] K. Grill-Spector, Z. Kourtzi, and N. Kanwisher. The lateral occipital complex and its role in object recognition. Vision Research, 41(10-11):1409-1422, 2001.

[79] K. Grill-Spector, T. Kushnir, S. Edelman, G. Avidan, Y. Itzchak, and R. Malach. Differential processing of objects under various viewing conditions in the human lateral occipital complex. Neuron, 24(1):187-203, September 1999.

[80] K. Grill-Spector, T. Kushnir, T. Hendler, S. Edelman, Y. Itzchak, and R. Malach. A sequence of object-processing stages revealed by fMRI in the human occipital lobe. Human Brain Mapping, 6(4):316-328, 1998.

[81] K. Grill-Spector, T. Kushnir, T. Hendler, and R. Malach. The dynamics of objectselective activation correlate with recognition performance in humans. Nature Neuroscience, 3(8):837-843, August 2000.

[82] A. F. Hamilton and S. T. Grafton. Goal representation in human anterior intraparietal sulcus. Journal of Neuroscience, 26(4):1133-1137, January 2006.

[83] J. Hegdé and D. C. Van Essen. Stimulus dependence of disparity coding in primate visual area V4. Journal of Neurophysiology, 93(1):620-626, January 2005.

[84] M. Himmelbach and H.-O. Karnath. Dorsal and ventral stream interaction: contributions from optic ataxia. The Journal of Cognitive Neuroscience, 17(4):632-640, April 2005.

[85] D. A. Hinkle and C. E. Connor. Three-dimensional orientation tuning in macaque area V4. Nature Neuroscience, 5(7):665-670, 2002.

[86] G. K. Humphrey, M. A. Goodale, L. S. Jakobson, and P. Servos. The role of surface information in object recognition: studies of a visual form agnosic and normal subjects. Perception, 23(12):1457-1481, 1994.

[87] K. H. James, G. K. Humphrey, and M. A. Goodale. Manipulating and recognizing virtual objects: where the action is. Canadian Journal of Experimental Psychology, 55(2):111-120, June 2001.

[88] T. W. James, J. C. Culham, G. K. Humphrey, A. D. Milner, and M. A. Goodale. Ventral occipital lesions impair object recognition but not object-directed grasping: an fMRI study. Brain, 126(Pt 11):2463-2475, November 2003.

[89] T.W. James, G.K. Humphrey, J.S. Gati, R.S. Menon, and M.A. Goodale. Differential effects of viewpoint on object-driven activation in dorsal and ventral streams. Neuron, 35(4):793-801, August 2002.

[90] L. Jäncke, A. Kleinschmidt, S. Mirzazade, N. J. Shah, and H. J. Freund. The role of the inferior parietal cortex in linking the tactile perception and manual construction of object shapes. Cerebral Cortex, 11(2):114-121, February 2001.

[91] P. Janssen, R. Vogels, and G. A. Orban. Selectivity for 3D shape that reveals distinct areas within macaque inferior temporal cortex. Science, 288(5473):2054-2056, June 2000.

[92] M. Jeannerod. The cognitive neuroscience of action. Blackwell, 1997.

[93] M. Jeannerod, M. A. Arbib, G. Rizzolatti, and H. Sakata. Grasping objects: the cortical mechanisms of visuomotor transformation. Trends in Neurosciences, 18(7):314320, July 1995.

[94] M. Jeannerod and P. Jacob. Visual cognition: a new look at the two-visual systems 
model. Neuropsychologia, 43(2):301-312, 2005.

[95] S. H. Johnson-Frey, R. Newman-Norlund, and S. T. Grafton. A distributed left hemisphere network active during planning of everyday tool use skills. Cerebral Cortex, 15(6):681-695, June 2005.

[96] J. F. Kalaska, P. Cisek, and N. Gosselin-Kessiby. Mechanisms of selection and guidance of reaching movements in the parietal lobe. Advances in Neurology, 93:97-119, 2003.

[97] N. Katsuyama, T. Naganuma, H. Sakata, and M. Taira. Coding of 3D curvature in the parietal cortex (area CIP) of macaque monkey. In International Symposium on Autonomous Minirobots for Research and Edutainment, pages 181-186, 2005.

[98] M. Kawato, T. Kuroda, H. Imamizu, E. Nakano, S. Miyauchi, and T. Yoshioka. Internal forward models in the cerebellum: fMRI study on grip force and load force coupling. Progress in Brain Research, 142:171-188, 2003.

[99] C. S. Konen and S. Kastner. Two hierarchically organized neural systems for object information in human visual cortex. Nat Neurosci, 11(2):224-231, Feb 2008.

[100] Z. Kourtzi, M. Erb, W. Grodd, and H. H. Bülthoff. Representation of the perceived 3-D object shape in the human lateral occipital complex. Cerebral Cortex, 13(9):911920, September 2003.

[101] Z. Kourtzi and E. Huberle. Spatiotemporal characteristics of form analysis in the human visual cortex revealed by rapid event-related fMRI adaptation. Neuroimage, 28(2):440-452, November 2005.

[102] Z. Kourtzi and N. Kanwisher. Cortical regions involved in perceiving object shape. Journal of Neuroscience, 20(9):3310-3318, May 2000.

[103] Z. Kourtzi and N. Kanwisher. Representation of perceived object shape by the human lateral occipital complex. Science, 293(5534):1506-1509, August 2001.

[104] M. A. Lebedev, A. Messinger, J. D. Kralik, and S. P. Wise. Representation of attended versus remembered locations in prefrontal cortex. PLoS Biology, 2(11):e365, November 2004.

[105] T. S. Lee. Computations in the early visual cortex. Journal of Physiology - Paris, 97(2-3):121-139, 2003.

[106] J. W. Lewis and D. C. Van Essen. Corticocortical connections of visual, sensorimotor, and multimodal processing areas in the parietal lobe of the macaque monkey. Journal of Comparative Neurology, 428(1):112-137, December 2000.

[107] G. Luppino, A. Murata, P. Govoni, and M. Matelli. Largely segregated parietofrontal connections linking rostral intraparietal cortex (areas AIP and VIP) and the ventral premotor cortex (areas F5 and F4). Experimental Brain Research, 128(1-2):181-187, September 1999.

[108] G. Luppino and G. Rizzolatti. The organization of the frontal motor cortex. News in Physiological Sciences, 15:219-224, October 2000.

[109] R. Malach, I. Levy, and U. Hasson. The topography of high-order human object areas. Trends in Cognitive Sciences, 6(4):176-184, April 2002.

[110] J. H. Maunsell. Functional visual streams. Current Opinion in Neurobiology, 2(4):506510, August 1992.

[111] A. D. Milner, H. C. Dijkerman, L. Pisella, R. D. McIntosh, C. Tilikete, A. Vighetto, and Y. Rossetti. Grasping the past. Delay can improve visuomotor performance. Current Biology, 11(23):1896-1901, November 2001. 
[112] A. D. Milner and M. A. Goodale. Visual pathways to perception and action. Progress in Brain Research, 95:317-337, 1993.

[113] A. D. Milner and M. A. Goodale. The visual brain in action. Oxford University Press, 1995.

[114] A. D. Milner, Y. Paulignan, H. C. Dijkerman, F. Michel, and M. Jeannerod. A paradoxical improvement of misreaching in optic ataxia: new evidence for two separate neural systems for visual localization. Proceedings of the Royal Society B - Biological Sciences, 266(1434):2225-2229, November 1999.

[115] A. D. Milner, D. I. Perrett, R. S. Johnston, P. J. Benson, T. R. Jordan, D. W. Heeley, D. Bettucci, F. Mortara, R. Mutani, and E. Terazzi. Perception and action in 'visual form agnosia'. Brain, 114 ( Pt 1B):405-428, Feb 1991.

[116] C. Moore and S.A. Engel. Neural response to the perception of volume in the lateral occipital complex. Neuron, 29(1):277-286, 2001.

[117] A. Murata, L. Fadiga, L. Fogassi, V. Gallese, V. Raos, and G. Rizzolatti. Object representation in the ventral premotor cortex (area F5) of the monkey. Journal of Neurophysiology, 78(4):2226-2230, October 1997.

[118] A. Murata, V. Gallese, G. Luppino, M. Kaseda, and H. Sakata. Selectivity for the shape, size, and orientation of objects for grasping in neurons of monkey parietal area AIP. Journal of Neurophysiology, 83(5):2580-2601, May 2000.

[119] T. Naganuma, I. Nose, K. Inoue, A. Takemoto, N. Katsuyama, and M. Taira. Information processing of geometrical features of a surface based on binocular disparity cues: an fMRI study. Neuroscience Research, 51(2):147-155, February 2005.

[120] S. D. Newman, R. L. Klatzky, S. J. Lederman, and M. A. Just. Imagining material versus geometric properties of objects: an fMRI study. Cognitive Brain Research, 23(23):235-246, May 2005.

[121] J. D. Nguyenkim and G. C. DeAngelis. Disparity-based coding of three-dimensional surface orientation by macaque middle temporal neurons. Journal of Neuroscience, 23(18):7117-7128, August 2003.

[122] G. A. Orban, K. Claeys, K. Nelissen, R. Smans, S. Sunaert, J. T. Todd, C. Wardak, J.B. Durand, and W. Vanduffel. Mapping the parietal cortex of human and non-human primates. Neuropsychologia, 44(13):2647-2667, 2006.

[123] G. A. Orban, D. Fize, H. Peuskens, K. Denys, K. Nelissen, S. Sunaert, J. Todd, and W. Vanduffel. Similarities and differences in motion processing between the human and macaque brain: evidence from fMRI. Neuropsychologia, 41(13):1757-1768, 2003.

[124] G. A. Orban, P. Janssen, and R. Vogels. Extracting 3D structure from disparity. Trends in Neurosciences, 29(8):466-473, August 2006.

[125] A. J. Parker. From binocular disparity to the perception of stereoscopic depth. In L. M. Chalupa and J. S. Werner, editors, The visual neurosciences, chapter 49, pages 779-792. MIT Press, Cambridge, MA, 2004.

[126] R. E. Passingham and I. Toni. Contrasting the dorsal and ventral visual systems: guidance of movement versus decision making. Neuroimage, 14(1 Pt 2):S125-S131, July 2001.

[127] G. F. Poggio, F. Gonzalez, and F. Krause. Stereoscopic mechanisms in monkey visual cortex: binocular correlation and disparity selectivity. Journal of Neuroscience, 8(12):4531-4550, December 1988. 
[128] N. Ramnani, I. Toni, R. E. Passingham, and P. Haggard. The cerebellum and parietal cortex play a specific role in coordination: a PET study. Neuroimage, 14(4):899-911, October 2001.

[129] V. Raos, M.-A. Umiltà, A. Murata, L. Fogassi, and V. Gallese. Functional properties of grasping-related neurons in the ventral premotor area F5 of the macaque monkey. Journal of Neurophysiology, 95(2):709-729, February 2006.

[130] C. L. Reed, S. Shoham, and E. Halgren. Neural substrates of tactile object recognition: an fMRI study. Human Brain Mapping, 21(4):236-246, April 2004.

[131] N. J. Rice, R. D. McIntosh, I. Schindler, M. Mon-Williams, J.-F. Dmonet, and A. D. Milner. Intact automatic avoidance of obstacles in patients with visual form agnosia. Experimental Brain Research, 174(1):176-188, September 2006.

[132] N. J. Rice, E. Tunik, and S. T. Grafton. The anterior intraparietal sulcus mediates grasp execution, independent of requirement to update: new insights from transcranial magnetic stimulation. Journal of Neuroscience, 26(31):8176-8182, August 2006.

[133] G. Rizzolatti and M. A. Arbib. Language within our grasp. Trends in Neurosciences, 21:188-194, 1998.

[134] G. Rizzolatti, R. Camarda, L. Fogassi, M. Gentilucci, G. Luppino, and M. Matelli. Functional organization of inferior area 6 in the macaque monkey II. Area F5 and the control of distal movements. Experimental Brain Research, 71(3):491-507, 1988.

[135] G. Rizzolatti and L. Craighero. The mirror-neuron system. Annual Review of Neuroscience, 27:169-192, 2004.

[136] G. Rizzolatti, L. Fadiga, V. Gallese, and L. Fogassi. Premotor cortex and the recognition of motor actions. Cognitive Brain Research, 3(2):131-141, March 1996.

[137] G. Rizzolatti and G. Luppino. The cortical motor system. Neuron, 31(6):889-901, September 2001.

[138] G. Rizzolatti, G. Luppino, and M. Matelli. The organization of the cortical motor system: new concepts. Electroencephalography and Clinical Neurophysiology, 106(4):283296, April 1998.

[139] G. Rizzolatti and M. Matelli. Two different streams form the dorsal visual system: anatomy and functions. Experimental Brain Research, 153(2):146-157, November 2003.

[140] J. Y. Ro, D. Debowy, S. Ghosh, and E. P. Gardner. Depression of neuronal firing rates in somatosensory and posterior parietal cortex during object acquisition in a prehension task. Experimental Brain Research, 135(1):1-11, November 2000.

[141] P. E. Roland, B. O'Sullivan, and R. Kawashima. Shape and roughness activate different somatosensory areas in the human brain. Proceedings of the National Academy of Sciences USA, 95(6):3295-3300, March 1998.

[142] S. Rozzi, R. Calzavara, A. Belmalih, E. Borra, G. G. Gregoriou, M. Matelli, and G. Luppino. Cortical connections of the inferior parietal cortical convexity of the macaque monkey. Cerebral Cortex, 16(10):1389-1417, October 2006.

[143] M. F. S. Rushworth, T. E. J. Behrens, and H. Johansen-Berg. Connection patterns distinguish 3 regions of human parietal cortex. Cerebral Cortex, 16(10):1418-1430, October 2006.

[144] R. M. Rutschmann and M. W. Greenlee. Bold response in dorsal areas varies with relative disparity level. Neuroreport, 15(4):615-619, March 2004. 
[145] H. Sakata, M. Taira, M. Kusunoki, A. Murata, and Y. Tanaka. The TINS lecture. The parietal association cortex in depth perception and visual control of hand action. Trends in Neurosciences, 20(8):350-357, August 1997.

[146] H. Sakata, M. Taira, M. Kusunoki, A. Murata, Y. Tanaka, and K. Tsutsui. Neural coding of 3D features of objects for hand action in the parietal cortex of the monkey. Philosophical Transactions of the Royal Society B: Biological Sciences, 353(1373):13631373, August 1998.

[147] H. Sakata, M. Taira, M. Kusunoki, A. Murata, K. Tsutsui, Y. Tanaka, W. N. Shein, and Y. Miyashita. Neural representation of three-dimensional features of manipulation objects with stereopsis. Experimental Brain Research, 128(1-2):160-169, September 1999.

[148] H. Sakata, M. Taira, A. Murata, and S. Mine. Neural mechanisms of visual guidance of hand action in the parietal cortex of the monkey. Cerebral Cortex, 5(5):429-438, 1995.

[149] H. Sakata, K. I. Tsutsui, and M. Taira. Toward an understanding of the neural processing for 3D shape perception. Neuropsychologia, 43(2):151-161, 2005.

[150] T. Schenk, A. Ellison, N. Rice, and A. D. Milner. The role of V5/MT+ in the control of catching movements: an rTMS study. Neuropsychologia, 43(2):189-198, 2005.

[151] H. Scherberger and R.A. Anderson. Sensorimotor transformation in the posterior parietal cortex. In L. M. Chalupa and J. S. Werner, editors, The visual neurosciences. MIT Press, 2004.

[152] E. Shikata, F. Hamzei, V. Glauche, R. Knab, C. Dettmers, C. Weiller, and C. Büchel. Surface orientation discrimination activates caudal and anterior intraparietal sulcus in humans: an event-related fMRI study. Journal of Neurophysiology, 85(3):1309-1314, 2001.

[153] E. Shikata, F. Hamzei, V. Glauche, M. Koch, C. Weiller, F. Binkofski, and C. Büchel. Functional properties and interaction of the anterior and posterior intraparietal areas in humans. European Journal of Neuroscience, 17(5):1105-1110, March 2003.

[154] E. Shikata, Y. Tanaka, H. Nakamura, M. Taira, and H. Sakata. Selectivity of the parietal visual neurones in 3D orientation of surface of stereoscopic stimuli. Neuroreport, 7(14):2389-2394, October 1996.

[155] L. Shmuelof and E. Zohary. Dissociation between ventral and dorsal fMRI activation during object and action recognition. Neuron, 47(3):457-470, August 2005.

[156] A. Singhal, J. C. Culham, E. Chinellato, and M. A. Goodale. Dual-task interference is greater in delayed grasping than in visually guided grasping. J. Vis., 7(5):1-12, 5 2007.

[157] T. Sugio, T. Inui, K. Matsuo, M. Matsuzawa, G. H. Glover, and T. Nakai. The role of the posterior parietal cortex in human object recognition: a functional magnetic resonance imaging study. Neuroscience Letters, 276(1):45-48, November 1999.

[158] T. Sugio, K. Ogawa, and T. Inui. Multiple action representations of familiar objects with handles: An fMRI study. In European Conference on Visual Perception, 2003.

[159] M. Taira, S. Mine, A. P. Georgopoulos, A. Murata, and H. Sakata. Parietal cortex neurons of the monkey related to the visual guidance of hand movement. Experimental Brain Research, 83(1):29-36, 1990.

[160] M. Taira, K.-I. Tsutsui, M. Jiang, K. Yara, and H. Sakata. Parietal neurons represent 
surface orientation from the gradient of binocular disparity. Journal of Neurophysiology, 83(5):3140-3146, May 2000.

[161] M. J. Tarr and H. H. Bülthoff. Image-based object recognition in man, monkey and machine. Cognition, 67(1-2):1-20, July 1998.

[162] J. T. Todd. The visual perception of 3D shape. Trends in Cognitive Sciences, 8(3):115121, March 2004.

[163] R. B. H. Tootell, J. D. Mendola, N. K. Hadjikhani, P. J. Ledden, A. K. Liu, J. B. Reppas, M. I. Sereno, and A. M. Dale. Functional analysis of V3A and related areas in human visual cortex. Journal of Neuroscience, 17(18):7060-7078, September 1997.

[164] R. B. H. Tootell, D. Y. Tsao, and W. Vanduffel. Neuroimaging weighs in: humans meet macaques in "primate" visual cortex. Journal of Neuroscience, 23(10):3981-3989, May 2003.

[165] D. Y. Tsao, W. Vanduffel, Y. Sasaki, D. Fize, T. A. Knutsen, J. B. Mandeville, L. L. Wald, A. M. Dale, B. R. Rosen, D. C. Van Essen, M. S. Livingstone, G. A. Orban, and R. B. H. Tootell. Stereopsis activates V3A and caudal intraparietal areas in macaques and humans. Neuron, 39(3):555-568, July 2003.

[166] K.-I. Tsutsui, M. Jiang, H. Sakata, and M. Taira. Short-term memory and perceptual decision for three-dimensional visual features in the caudal intraparietal sulcus (area CIP). Journal of Neuroscience, 23(13):5486-5495, July 2003.

[167] K.-I. Tsutsui, M. Jiang, K. Yara, H. Sakata, and M. Taira. Integration of perspective and disparity cues in surface-orientation-selective neurons of area CIP. Journal of Neurophysiology, 86(6):2856-2867, December 2001.

[168] K.-I. Tsutsui, M. Taira, and H. Sakata. Neural mechanisms of three-dimensional vision. Neuroscience Research, 51(3):221-229, March 2005.

[169] E. Tunik, S. H. Frey, and S. T. Grafton. Virtual lesions of the anterior intraparietal area disrupt goal-dependent on-line adjustments of grasp. Nature Neuroscience, 8(4):505-511, April 2005.

[170] E. Tunik, N. J. Rice, A. Hamilton, and S. T. Grafton. Beyond grasping: representation of action in human anterior intraparietal sulcus. Neuroimage, 36 Suppl 2:T77-T86, 2007.

[171] L.G. Ungerleider and M. Mishkin. Two cortical visual systems. In D.J. Ingle, M.A. Goodale, and R.J.W. Mansfield, editors, Analysis of visual behavior, pages 549-586. MIT Press, 1982.

[172] K. F. Valyear, C. Cavina-Pratesi, A. J. Stiglick, and J. C. Culham. Does tool-related fMRI activity within the intraparietal sulcus reflect the plan to grasp? Neuroimage, 36 Suppl 2:T94-T108, 2007.

[173] K. F. Valyear, J. C. Culham, N. Sharif, D. Westwood, and M. A. Goodale. A double dissociation between sensitivity to changes in object identity and object orientation in the ventral and dorsal visual streams: A human fMRI study. Neuropsychologia, 44(2):218-228, 2006.

[174] D. C. Van Essen, J. W. Lewis, H. A. Drury, N. Hadjikhani, R. B. Tootell, M. Bakircioglu, and M. I. Miller. Mapping visual cortex in monkeys and humans using surfacebased atlases. Vision Research, 41(10-11):1359-1378, 2001.

[175] W. Vanduffel, D. Fize, H. Peuskens, K. Denys, S. Sunaert, J. T. Todd, and G. A. Orban. Extracting 3D from motion: differences in human and monkey intraparietal 
cortex. Science, 298(5592):413-415, October 2002.

[176] S. Vanni, M. Dojat, J. Warnking, C. Delon-Martin, C. Segebarth, and J. Bullier. Timing of interactions across the visual field in the human cortex. Neuroimage, 21(3):818 828, March 2004.

[177] M. J. Webster, J. Bachevalier, and L. G. Ungerleider. Connections of inferior temporal areas TEO and TE with parietal and frontal cortex in macaque monkeys. Cerebral Cortex, 4(5):470-483, 1994.

[178] A. E. Welchman, A. Deubelius, V. Conrad, H. H. Bülthoff, and Z. Kourtzi. 3D shape perception from combined depth cues in human visual cortex. Nature Neuroscience, 8(6):820-827, June 2005.

[179] D. A. Westwood, J. Danckert, P. Servos, and M. A. Goodale. Grasping twodimensional images and three-dimensional objects in visual-form agnosia. Experimental Brain Research, 144(2):262-267, May 2002.

[180] D. A. Westwood and M. A. Goodale. A haptic size-contrast illusion affects size perception but not grasping. Experimental Brain Research, 153(2):253-259, November 2003.

[181] J.H.G. Williams, A. Whiten, T. Suddendorf, and D.I. Perrett. Imitation, mirror neurons and autism. Neuroscience and Biobehavioural Review, 25:287-295, 2001.

[182] A. Winkler, C. E. Wright, and C. Chubb. Dissociating the functions of visual pathways using equisalient stimuli. Journal of Vision, 5(8):362, 2005. 